\title{
Effects of putrescine supplementation on growth performance, blood lipids and immune response in broiler chickens fed methionine deficient diet
}

\begin{abstract}
The polyamines including putrescine (PUT), spemidine (SPD) and spermine (SPM) have been shown to play an important role in basic cellular processes. Methionine (Met) and arginine are required for polyamine biosynthesis. This experiment was carried out to study the effects of Met deficient diet with or without supplemental PUT on the performance, blood lipids and glucose and humoral immunity in broiler chickens. A total of 192 day-old chicks were allocated into factorial arrangement $(2 \times 2)$ of four dietary treatments including two Met levels (Normal and Low) and two PUT levels (0 and 0.03\%). Body weight and feed intake were measured weekly. At the age of $28 \mathrm{~d}, 10$ birds per treatment were challenged with infectious bursal disease vaccine orally $(10$ dosage/bird, $2 \mathrm{ml})$. Blood samples of the challenged birds were collected for antibody determination and white blood cell count. Blood cholesterol, triglyceride, glucose, hematocrit and protein of non-challenged birds were measured at the ages of 24, 33 and 40 d. Low Met decreased body weight gain, protein efficiency ratio and feed conversion ratio $(\mathrm{P}<0.05)$. In grower period, energy efficiency ratio was improved by PUT supplementation in the chicks fed with normal Met level. Feed conversion ratio was badly affected $(\mathrm{P}<0.05)$ by low Met during grower period. PUT decreased body weight gain of chickens fed low Met significantly $(\mathrm{P}<0.05)$, indicating the importance of adequate Met level when high dietary PUT is offered. Met deficient diet decreased plasma protein level significantly $(\mathrm{P}<0.05)$. Five days post challenged, PUT supplementation elevated antibody level significantly $(\mathrm{P}<0.05)$ in the chicks fed normal level of Met. However, Met deficient diet in challenged chicks, caused significantly $(\mathrm{P}<0.05)$ lower monocyte ratio. In conclusion, growth performance declined due to Met deficiency, particularly during starter period (0-21 d) and when dietary PUT was supplemented. PUT showed the potential to increase blood antibody level and better dietary energy efficiency ratio.
\end{abstract}

Keyword: Polyamines; Broiler chickens; Performance; Antibody; Blood lipids 